\begin{tabular}{|c|l|}
\hline Title & Opto-thermophoretic separation and trapping of plasmonic nanoparticles \\
\hline Author(s) & Setoura, Kenji; Tsuji, Tetsuro; Ito, Syoji; Kawano, Satoy uki; Miy asaka, Hiroshi \\
\hline Citation & $\begin{array}{l}\text { Nanoscale, 11(44), 21093-21102 } \\
\text { https://doi.org/10.1039/c9nro5052c }\end{array}$ \\
\hline Issue Date & 2019-11-28 \\
\hline Doc URL & http://hdl.handle.net/2115/79878 \\
\hline Type & article (author version) \\
\hline File Information & setoura.pdf \\
\hline
\end{tabular}

Instructions for use 


\section{Opto-Thermophoretic Separation and Trapping of Plasmonic Nanoparticles}

Kenji Setoura, ${ }^{*},+,+$ Tetsuro Tsuji, ${ }^{+, \#}$ Syoji Ito, ${ }^{*}{ }^{+}$Satoyuki Kawano, ${ }^{\dagger}$ and Hiroshi Miyasaka ${ }^{\dagger}$

${ }^{\dagger}$ Graduate School of Engineering Science, Osaka University, Toyonaka, Osaka 560-8531, Japan

${ }^{\ddagger}$ Research Institute for Electronic Science, Hokkaido University, Sapporo 001-0020, Japan

KEYWORDS: optical trapping; thermophoresis; plasmonic nanoparticles; gold nanoshells; nanoparticle separation

*Electronic supplementary information (ESI) is available 
Graphical abstract

\section{Trapping \& Separation}
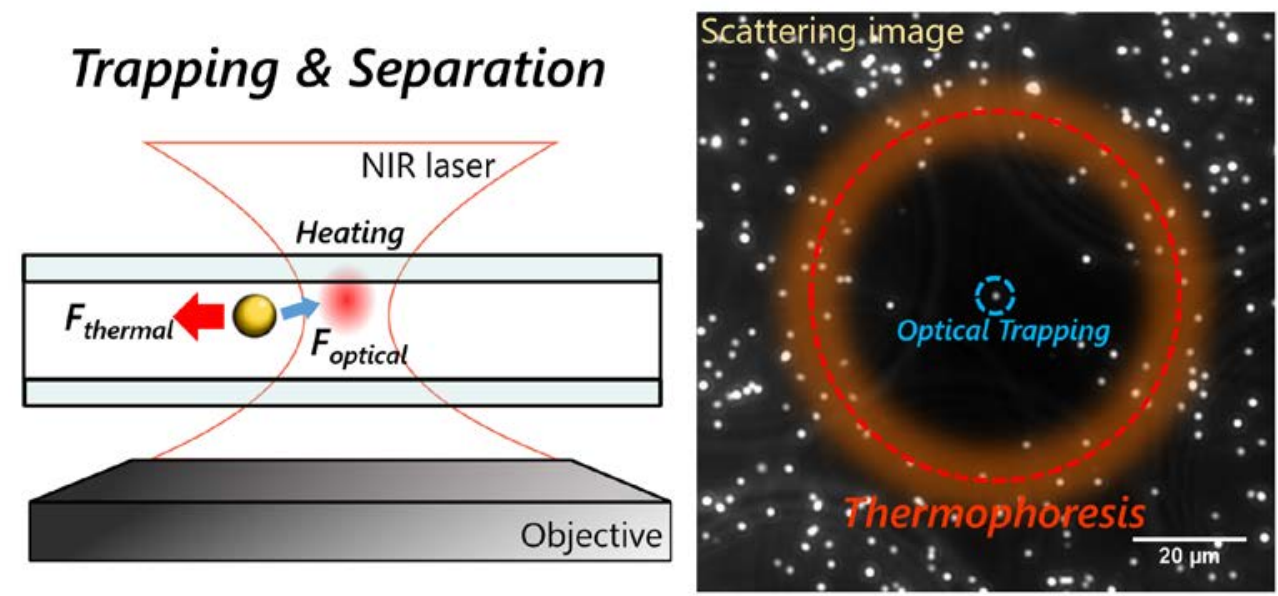

We have succeeded in the selective trapping of single gold nanoshells with specific sizes and sweeping others out completely, only by irradiating the dense colloidal suspension of gold nanoshells with a focused near infrared continuous-wave laser. 
ABSTRACT. Optical tweezers are powerful tools to trap, transport, and analyse individual nano-objects at dilute concentrations. However, it is still challenging to manipulate isolated single nano-objects in dense target environments with various kinds of materials, such as in living cells and mixtures of nanocolloids. In the present work, we have succeeded in the selective trapping of a few gold nanoshells with specific sizes and sweeping others out completely, only by irradiating the dense colloidal suspension of gold nanoshells with a focused near infrared continuous-wave (CW) laser. This was achieved by an interplay between optical gradient- and thermophoretic forces: while the gradient force traps the targets at the focal spot, the thermophoretic force pushes others out from the focal spot. The distance between the trapped targets and the separated others was longer than $20 \mu \mathrm{m}$, allowing us to measure plasmonic scattering spectra of the trapped targets at a single-nanoparticle level. The present method paves a way for manipulating and analysing single nano-objects in dense mixtures of targets and various kinds of materials. 
TEXT. Capturing, transporting, and analysing specific single nano-objects in dense environments with various kinds of materials, such as in living cells, are one of the important methods in nanoscience and nanotechnology. Optical tweezers are promising tools for trapping nano-objects to evaluate their mechanical responses and optical properties in a condensed phase., ${ }^{1,2}$ Current progress on the optical manipulation techniques enables selective transportation or trapping of quantum dots, ${ }^{3,4}$ carbon nanotubes, ${ }^{5}$ and plasmonic anisotropic nanoparticles. ${ }^{6}$ These methods are mainly based on the difference in their optical properties depending on materials, sizes, and shapes. It is, however, difficult to trap "specific" and "single" nano-objects with the optical tweezers in the dense environments, because an optical gradient force indiscriminately attracts any tiny objects with large polarizabilities. Accordingly, dilute concentrations are generally needed for analysing single nano-objects. ${ }^{7,8}$ This drawback limits practical applications of the optical tweezers, although several elegant demonstrations have been made for biological specimens at single-molecule levels. ${ }^{9,10}$

Here, we have introduced an additional force counterbalancing to the optical gradient force, to pave the way for the selective trapping of desired single nano-objects and the spatial separation of undesired others. The counterbalancing force employed in the present study is due to thermophoresis. ${ }^{11,12}$ Both the optical trapping and the thermophoresis can be induced only by irradiating a colloidal solution with a focused near infrared (NIR) laser (Figure 1a). Photon pressure of the NIR laser focused with a microscope objective acts as attractive force to the nanoparticle and traps it at the focal spot. ${ }^{1}$ At the same time, the NIR laser excites vibrational transitions of solvent molecules, resulting in local temperature elevation. ${ }^{13}$ This photothermal effect drives the thermophoresis as repulsive force, which sweeps the nanoparticles out of trap from hot to cold. ${ }^{14-17}$ By adjusting the ratios of the attractive- and the repulsive forces acting on 
the nanocolloids, the following three modes on optical manipulation will be available: i) optical trapping mode entirely governed by the gradient force, ii) thermophoretic depletion mode dominated by the thermophoresis, and iii) selective trapping and separation mode based on the interplay of both contributions.

With this method, larger nanoparticles will be selectively trapped by the optical gradient force while smaller nanoparticles will be swept out away by the thermophoresis in case of ordinary nanocolloids such as silica or polystyrene nanoparticles. This is because the polarizability of a Rayleigh particle increases with the cube of its radius, ${ }^{1}$ whereas the thermophoretic force is less sensitive to the particle size. ${ }^{18}$ On the other hand, several optical manipulation techniques for the separation purpose have been developed until now without any repulsive force. For instance, Misawa et al. demonstrated that polystyrene microspheres with a diameter of $1.0 \mu \mathrm{m}$ can be selectively trapped in diffraction patterns of the focused CW laser in a mixture of colloidal solution with diameters of $250 \mathrm{~nm}$ and $1.0 \mu \mathrm{m} .{ }^{19}$ MacDonald et al. demonstrated that a mixed colloidal solution of silica and polymer microspheres with same diameter can be separated and sorted based on the difference in their refractive indices in a microfluidic chamber under irradiation of an optical lattice. ${ }^{20}$ However, selective trapping and separation of smaller nanoparticles (or nanoparticles with low refractive indices) in a mixture of colloidal solutions is generally difficult because of a decrease in the optical gradient force. The realization of the above idea would provide a new methodology for selection and analysis of smaller nano-objects in the mixture of nanocolloids.

To demonstrate this, the aqueous solution of gold nanoshells (Au NSs) was used as a specimen in the present study. The Au NS is a core/shell type nanoparticle consisting of a silica core and a gold shell with a few tens of nanometers in thickness, which shows localized surface 
plasmon resonance (LSPR) in the NIR wavelength region. ${ }^{21}$ In general, silica nanoparticles exhibit active thermophoretic motions, ${ }^{22,23}$ but the optical gradient force acting on them are relatively small because of their small polarizabilities. ${ }^{24}$ In contrast, gold nanoparticles (Au NPs) can be easily trapped owing to their huge polarizabilities, ${ }^{25}$ whereas are insensitive to the thermophoresis due to the extremely high thermal conductivity of gold. ${ }^{16,26}$ In contrast to these materials, the $\mathrm{Au}$ NSs have fine plasmonic optical properties and intermediate thermal conductivities between silica and gold, so would be ideal nano-objects to demonstrate the present opto-thermophoretic manipulation (Figure 1b). Actually, we have observed that larger Au NSs with thinner shells were completely swept away by the thermophoresis, while a few smaller Au NSs with thicker shells were selectively trapped at the focal spot owing to an opto-thermal property of the Au NS depending on both an entire nanoshells volume and a core shell ratio. Sizes of the smaller ones were successfully evaluated with scattering micro-spectroscopy, because the larger scatterers were eliminated from the focal spot of the objective lens. The present method opens up pathways to selectively manipulate and analyse single nano-objects in dense environments. 


\section{Results and Discussion.}

Materials and Methods. The aqueous solution of Au NSs with diameters of about $240 \mathrm{~nm}$ (nanoComposix) was used as received. The average core diameter and shell thickness were 197 $\mathrm{nm}$ and $22 \mathrm{~nm}$, respectively. The surfaces of the Au NSs treated with lipoic acids are negatively charged. To prepare a thin sample chamber for observation with an optical microscope, $2 \mu \mathrm{L}$ of the aqueous colloidal solution was sandwiched between two glass coverslips (borosilicate glass, $24 \times 32 \times 0.17 \mathrm{~mm}$, Matsunami). The chamber was sealed with vacuum grease (HIVAC-G, ShinEtsu Silicones) to prevent the leaking of the colloidal solution. The thickness of the liquid layer was ca. $5 \mu \mathrm{m}$; the size was small enough to suppress natural convection and enabled us to observe the thermophoresis. ${ }^{26}$

Experiments were performed under an inverted optical microscope (IX73, Olympus). Details of the optical setup are shown in Figure S1 in Electronic Supplementary Information (ESI). In brief, dark field illumination was used to acquire scattering images of the Au NSs. For the simultaneous induction of the optical trapping and the thermophoresis, the sample was irradiated with a CW laser at a wavelength of $1475 \mathrm{~nm}$ through a microscope objective $(50 \times, \mathrm{NA}=0.55$, Olympus). Overtone vibrational transitions arising from $\mathrm{OH}$ bonds of water molecules in this wavelength region results in efficient photothermal heating and the induction of the thermophoresis.

Laser spot diameters are most important parameters in this experiment. To vary laser spot diameter at the focal plane of the objective, an inter-lens distance of a pair of plano-convex lenses was changed; note that the focal plane was fixed at an interface between the upper coverslip and the colloidal solution. We measured the intensity profile of the focused NIR laser by irradiating a thin film consists in microspheres of IR phosphors (IRSPG-5G, LDP LLC) to evaluate the laser 
spot diameters (see ESI S2). In the optical tweezers, the gradient force $\left(\mathbf{F}_{\text {grad }}\right)$ attracting nanoparticles is a function of the polarizability $(\alpha)$ of nanoparticles and the spatial gradient of the light intensity $\left(\nabla|E|^{2}\right){ }^{2}$ On the other hand, the thermophoretic force $\left(\mathbf{F}_{\text {Tphore }}\right)$ is proportional to a temperature gradient $(\nabla T) .{ }^{11,12}$ In the case of laser heating, the $\nabla T\left[\mathrm{~K} \mathrm{~m}^{-1}\right]$ is a function of both the laser spot diameter $[\mu \mathrm{m}]$ and the laser power $[\mathrm{mW}]$. However, the $\nabla T$ exhibits small dependence on the laser spot diameter compared to that for the $\nabla|E|^{2}$, because of diffusive nature of heat conduction. As a consequence, $\mathbf{F}_{\text {grad }}$ is more sensitive to changes in the laser spot diameter compared with $\mathbf{F}_{\text {Tphore }}$. Therefore, a ratio between $\mathbf{F}_{\text {grad }}$ and $\mathbf{F}_{\text {Tphore }}$ acting on the Au NSs is strongly dependent on the laser spot diameter, resulting in different photo-mechanical responses of AuNSs: the optical trapping, the thermophoretic depletion, or the simultaneous separation and trapping depending on photo-irradiation condition.

Optical trapping of gold nanoshells.

First, we examined the optical trapping of individual Au NSs with the tightly focused NIR laser. The spot diameter was $1.5 \mu \mathrm{m}$ in full width at half-maximum (fwhm) for $X$-coordinate. In all experiments, the focal plane of the objective was fixed at an interface between the upper coverslip and the colloidal solution. Figure 2a shows the scattering image of a single Au NS under trapping at a power density of $4.3 \times 10^{5} \mathrm{~W} \mathrm{~cm}^{-2}$ (see Supplementary Movie 1). Note that in the present paper, the power density refers an average power density in a $1 / \mathrm{e}^{2}$ spot diameter. ${ }^{27}$ Stable trapping was continued more than a few minutes, and the Au NS was released when the NIR laser was turned off. To verify this trapping behavior is due to the optical gradient force, we analysed the Brownian dynamics of the trapped Au NS. Figure 2b shows the trajectory of the Brownian motion of the Au NS, as determined by a localization method 
based on the 2D Gaussian fittings of the scattering images. ${ }^{28}$ As is clearly shown in this figure, positions of the Au NS were distributed around the focal spot of the NIR laser (at $X$ and $Y=0 \mathrm{~nm}$ ). For quantitative analyses, the time course and positional histogram of the Au NS along $X$ coordinate are plotted in Figure 2c and -d, respectively. From these results, it was confirmed that the Brownian motion of the Au NS was spatiotemporally confined around the position of $X=0$ nm. Markedly, the histogram shown in Figure 2d was successfully fitted with a Gaussian function (1/e radius: $84 \mathrm{~nm}$ ), indicating that the Au NS was trapped in a quasi-harmonic potential created by the gradient force. ${ }^{28}$

Moreover, the elliptical position distributions shown in Figure $2 \mathrm{~b}$ also indicate that the gradient force is the main origin of the present trapping. The elongated shape along $Y$-axis can be ascribed to an elliptical intensity pattern for the transverse modes of the diode NIR laser. As already mentioned in the section of Materials and Methods, we measured the intensity profile of the focused NIR laser by irradiating a thin film consists in microspheres of IR phosphors; the aspect ratio $(Y / X)$ of the elliptical emission pattern was ca. 1.2 (see ESI S2). In the same manner as shown in Figure 2d, we evaluated the Gaussian width along $Y$-axis (1/e radius: $104 \mathrm{~nm}$ ) and obtained an aspect ratio of 1.2 on the positional fluctuation. The individual histograms of the $X$ and $Y$-positions are shown in ESI S3. From this agreement on the aspect ratios on the intensity and positions, one can conclude that the trapping behavior of the Au NS was entirely governed by the gradient force, and even with the elliptical laser beam, a temperature field shapes concentric distributions because of heat transfer, ${ }^{29}$ therefore a possibility of purely thermophoretic trapping can be safely excluded. ${ }^{30}$

To gain a deeper insight into the trapping behavior, we evaluated the positional fluctuations (Gaussian widths as 1/e radii) as the function of laser power density at the laser spot diameters of 
1.5 and $1.7 \mu \mathrm{m}$ (Figure 3). The depth of the trapping potential becomes shallow with an increase in the spot diameter. The power dependencies at both spot diameters were similar to those in conventional optical tweezers. ${ }^{31,32}$ These results indicate that the trapping behavior of the Au NS is mainly dominated by the gradient force, and no contribution arising from thermophoretic force under the condition with the tightly focused irradiation. In this section, we evaluated the stability of optical trapping with Gaussian widths of the fluctuations. In actuality, a trapping stiffness is the best describing parameter of the stability. However, we were unable to utilize this parameter due to the following two reasons. i) Experimental determination of the trapping stiffness requires a high temporal resolution of a few tens of microseconds overcoming the cutoff frequency of the optical trapping. ${ }^{31}$ In this study, a frame rate of the CMOS camera (typically $25 \mathrm{~Hz}$ ) was too slow. ii) To estimate the trapping stiffness from experimental results shown in Figure 2, one needs to perform Brownian dynamics simulations to numerically determine a depth of the trapping potential. ${ }^{28}$ In this study, however, this simulation was difficult to perform because it requires full parameters such as scattering/absorption forces of the Au NS, temperature of the Au NS, and temperature distribution around the Au NS.

At the end of this section, we discuss the local temperature increase in the focal spot. Generally, the laser heating induces not only thermophoresis, but also several phenomena such as the activation of Brownian motion of solvents, ${ }^{13}$ microbubble formation, ${ }^{33,34}$ Marangoni convection, ${ }^{35,36}$ and so forth. The absorption coefficient of water molecules at the wavelength of $1475 \mathrm{~nm}$ is about 200 times larger than that at $1064 \mathrm{~nm}$ which is the most commonly used laser wavelength for optical manipulation. ${ }^{37}$ Accordingly, the estimation of the local temperature is essential for quantitative discussion. The average temperature increase in the focal spot was estimated to be $50 \mathrm{~K}$ as a maximum by the calculation based on a $1 \mathrm{D}$ analytical heat conduction 
model consisting of a radial heat source in a water medium (see ESI S4). ${ }^{13}$ This temperature increase, however, does not induce microbubble formation and subsequent Marangoni convection. ${ }^{33,38}$ Hence, in the following sections, we will consider only the gradient- and the thermophoretic forces for laser-induced migration of Au NSs. Note that we employed the 1D radial heat conduction model to estimate the order of magnitude of temperature in the laser spot. As will be shown later, we performed full scale 2D numerical simulation on heat conduction including glass substrates, to clearly illustrate the temperature distribution around the Au NS.

Thermophoretic depletion of gold nanoshells.

In general, Au NPs are considered to be less sensitive to the thermophoresis because of their high thermal conductivity. ${ }^{16,26}$ Similarly, weaker thermophoretic force can be expected for Au NSs compared to that for silica nanoparticles with equivalent volumes. In this section, we discuss the thermophoretic mobilities of the Au NSs by introducing the experimental results on the sweeping of Au NSs with an unfocused irradiation.

Figure 4a shows a scattering image accumulated 100 frames under irradiation at a power density of $2.2 \times 10^{5} \mathrm{~W} \mathrm{~cm}^{-2}$ and a laser spot diameter of $3.6 \mu \mathrm{m}$ : an exposure and a frame rate were $0.3 \mathrm{~s}$ and $1 \mathrm{~Hz}$ for acquisition, respectively. The 100 images were acquired after reaching the steady-state. The Supplementary Movie 2 shows the depletion dynamics. It took 30 to 40 s to reach the steady-state. In Figure 4a, the brightness of the scattering image represents the concentration of the Au NSs. Several brightest spots with the size $<$ a few $\mu \mathrm{m}$ in the image are dusts on the coverslip. As clearly shown in this figure, a central region under the irradiation was dark. The absence of the NSs at the central region in the time-averaged image is due to laser-induced thermophoretic depletion, ${ }^{39,40}$ i.e., the evacuation of NSs from the hot place to the cold one. This 
figure indicated that the Au NSs are thermophoretically active owing to their intermediate thermal conductivity of metal and silica, although pure Au NPs with similar sizes did not show such depletion. ${ }^{16,26}$ The concentration of the Au NSs gradually increases with an increase in the distance from the laser spot. In addition, we assumed that optical scattering forces along $X$ - and $Y$-axis are minor contribution, because of the quite large area of the depletion compared with that of the laser spot.

To visualize the spatial distribution of the depletion more clearly, we plotted a radial intensity profile averaged over entire azimuthal directions from the focal spot in Figure 4b. At the spot diameter of $3.6 \mu \mathrm{m}$ (a red dotted line), the intensity at the center is almost zero. This indicates that Au NSs was not trapped in the laser spot during the image acquisition. Although the intensity starts to increase around at the radial distance of 10 to $20 \mu \mathrm{m}$, the spatial distribution is affected by the thermophoresis even at $40 \mu \mathrm{m}$. The black solid line in Figure $4 \mathrm{~b}$ shows an averaged profile at the same power density with the laser spot diameter of $2.5 \mu \mathrm{m}$. The spatial distributions of both conditions show similar profiles, but the intensity is higher for the spot diameter of $2.5 \mu \mathrm{m}$. This result indicates that decrease in the spot diameter results in an increase of the ratio of $\left|\mathbf{F}_{\text {grad }}\right| / \mid$

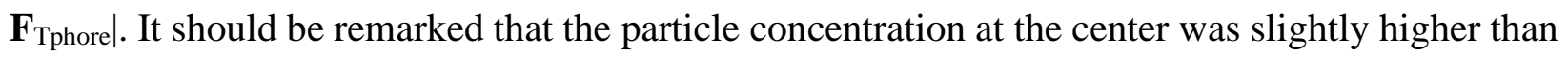
zero at the spot diameter of $2.5 \mu \mathrm{m}$, indicating that a few Au NSs could stay at the laser spot due to simultaneous induction of the selective optical trapping and the thermophoretic depletion.

Sorting of gold nanoshells. As a result of the optimization, we found an irradiation condition that a single Au NS was optically trapped and others were swept away from the laser spot at the power density of $3.2 \times 10^{5} \mathrm{~W} \mathrm{~cm}^{-2}$ and the spot diameter of $3.0 \mu \mathrm{m}$. Figure 5 a shows a 
scattering image of the simultaneous trapping and depletion (see Supplementary Movie 3). A single Au NS was successfully trapped at the center of the image, while others were swept away for $20 \mu \mathrm{m}$ from the center. Figure 5b shows the positions of the trapped Au NS obtained in the same manner as shown in Figure 2b. This elliptical shape again proves that the trapping behavior is dominated by the gradient force, although the fluctuation is doubled compared to that in Figure 2b because of an increase in laser spot diameter. Looking at Figure 5a and Supplementary Movie 3 carefully, we noticed that brighter Au NSs can only be located far from the laser spot. To analyse this interesting tendency, we evaluated the maximum brightness of each Au NS as the function of radial distance from the laser spot by means of the localization method, as shown in Figure 5c. Obviously, the Au NSs are sorted in order of brightness from dim to bright along the radial coordinate. In principle, a scattering intensity of a plasmonic nanoparticle increases with size. ${ }^{41}$ Hence, this sorting can be interpreted that larger Au NSs were pushed out by the thermophoresis, and smaller Au NSs got closer to the laser spot because they could pass through a potential barrier created by the thermophoresis. In addition, this interpretation can be reinforced by the following two facts; i) if the laser was initially focused onto the bright Au NS, it was always pushed away from the focal spot. The dim Au NS was successfully trapped when it was initially in the focal spot. ii) if the laser was focused at the random at the interface, only the dim Au NS came into the focal spot and was optically trapped within a few tens of seconds from the start of the irradiation. However, as already mentioned in the introductory part, the plasmonic optical properties of the Au NSs depend on both the core/shell ratio and the entire nanoshell's volume. ${ }^{21}$ Therefore, it is still unclear that which factors of the Au NS determines the behavior of the selective trapping and depletion. 
To elucidate the details of the factors, we performed 2D numerical simulations on the steady state heat conduction to evaluate the thermophoretic force acting on a Au NS at various core/shell ratios. Here we consider a realistic system consisting of a thin water layer of a thickness of $5 \mu \mathrm{m}$ sandwiched with two glass substrates of a thickness of $170 \mu \mathrm{m}$. A half circular heat source with a radius of $1.5 \mu \mathrm{m}$ is placed at the interface between the water and the glass substrate, because the NIR laser was focused onto the interface. This radius was determined from the experimental condition in Figure 5a. A single Au NS is placed $20 \mu \mathrm{m}$ away from the heat source in the water layer; this was empirically determined from the radial distance of the thermophoretic depletion in Figure 5c. In this simulation, optical phenomena and fluid convection are not considered. Accordingly, governing equations are Laplace and Poisson type heat conduction equations for water, glass substrates and Au NSs, respectively. ${ }^{42}$ The equations are given by, ${ }^{42}$

$$
\begin{gathered}
k \nabla^{2} T(X, Y)=Q(X, Y) \\
k \nabla^{2} T(X, Y)=0
\end{gathered}
$$

where $k$ is the thermal conductivity, $Q$ is the heat source. The origins of $X-Y$ coordinates are the center of the radial heat source. Thermal conductivities of $0.6,1.0$, and $314\left[\mathrm{~W} \mathrm{~m}^{-1} \mathrm{~K}^{-1}\right]$ were used for water, silica (both substrates and silica cores), and gold shells, respectively. Boundary conditions for heat flux at water-glass, water-gold, and silica-gold interfaces are continuity. If one needs to calculate temperature in the laser spot as a function of power density, the source term $Q$ should be exactly determined from net input energy and the absorption coefficient of water. ${ }^{29}$ 
However, these procedures are not necessary for the evaluation of the temperature difference $(\Delta T)$ at the surface of a Au NS exposed to the temperature gradient, and we can simply put a certain value for the $Q$ to gain the temperature of $343 \mathrm{~K}$ in the heat source (50 K as a temperature increase). This value is based on the temperature estimation shown in ESI S4. A constant temperature of 293 K was set to outer boundaries of the water layer and the glass substrates. The actual calculation was numerically performed with COMSOL multiphysics on the basis of a finite element method.

Figure 6a shows a 2D temperature distribution in the water layer and the glass substrates. The heat source is located around $X$ and $Y=0 \mu \mathrm{m}$, and the temperature is $343 \mathrm{~K}$. With increase in distance from the heat source, the temperature gradually decreases in proportion to the reciprocal of the distance. This is the typical temperature distribution being created by a point-like heat source in a homogeneous medium. ${ }^{43,44}$ The temperature gradient in Figure $6 \mathrm{a}$ is on the order of $10^{6} \mathrm{~K} \mathrm{~m}^{-}$ ${ }^{1}$ which is enough for the induction of the thermophoresis. ${ }^{22,23}$ What should be focused on here is, a tiny Au NS (a total diameter: $240 \mathrm{~nm}$, a core diameter: $200 \mathrm{~nm}$, and a shell thickness: $20 \mathrm{~nm}$ ) located at $X=20 \mu \mathrm{m}$ and $Y=-2.5 \mu \mathrm{m}$. To more clearly visualize the $\Delta T$, a magnified temperature distribution around the Au NS is shown in Figure 6b. Note that a color bar in Figure 6b represents an extremely narrow temperature range. The left hand side in Figure 6b is closer to the heat source, showing that the temperature is slightly higher than that at the right hand side. Even at the left and right edges of this temperature map, there is only the temperature difference of $1.0 \mathrm{~K}$, but this temperature gradient induces the thermophoresis of the Au NSs. As a driving force of the thermophoresis, the maximum $\Delta T$ at the surface of the Au NS is most important. In Figure 6b, the $\Delta T$ at the surface along $X$-axis is $2.1 \mathrm{mK}$. Although this value is still one-order larger than that of pure Au NPs with the equivalent size, we presumed that this is underestimation due to the unknown effective thermal conductivity of gold shells. The gold shell of Au NSs employed in this work 
consists in aggregated very tiny Au NPs. Therefore, these gold shells can be better modeled as a porous thin film rather than a continuum thin layer. For porous metals, it has been reported that a thermal conductivity may drop to $50 \%$ of the bulk value with void fraction. ${ }^{45}$ However, there have been no reports on the effective thermal conductivity of plasmonic gold shells. Hence, more realistic estimation is difficult at the present stage.

Next, we calculated the $\Delta T$ at various core/shell ratios for the Au NS with the total diameter of $240 \mathrm{~nm}$, of which value is similar with those of the experimentally used particles. Figure 6c shows the $\Delta T$ as the function of core diameter. In this figure, the Au NSs with smaller cores have thicker shells and the ones with larger cores have thinner shells, because the total nanoshell's diameter is fixed. This figure clearly shows that the $\Delta T$ increases with core diameter. This result can be interpreted as follows. The Au NS with large shell thickness (small core diameter) has large thermal conductivities owing to the thick Au shell resulting in smaller. In contrast, core-rich Au NSs have small thermal conductivities leading to large $\Delta T$, and are swept away by the thermophoresis. The core/shell ratio dependence in Figure 6c can clearly explain the trapping and sorting of the Au NSs observed in Figure 5c. On the basis of these temperature simulations, we calculated the thermophoretic forces acting on the Au NS and the drag force driven by the natural convection. As a result, the thermophoretic force was a few tens of $\mathrm{fN}$ at maximum, while the Stokes drag force was less than $4 \times 10^{-2} \mathrm{fN}$ (see ESI S5). These values support that the separation and the sorting of Au NSs are induced by the thermophoresis.

\section{Single particle manipulation and spectroscopic characterization.}


To clearly demonstrate selective trapping and the spectroscopic characterization of single nano-objects at dense concentrations, we prepared the mixture of Au NSs with different sizes. For this purpose, the aqueous solution of Au NSs with diameters about $140 \mathrm{~nm}$ (core diameters: ca. 80 $\mathrm{nm}$ and shell thickness: ca. $30 \mathrm{~nm}$, nanoComposix) was used as received. The resonance bands of plasmonic scattering are $650 \mathrm{~nm}$ and $1000 \mathrm{~nm}$ for the Au NSs with the diameters of $140 \mathrm{~nm}$ and $240 \mathrm{~nm}$, respectively. Hence, one can easily evaluate the sizes of the trapped ones with a conventional micro-spectroscopy system for visible wavelength regions. The thin sample chamber consisting of two coverslips was prepared in the same manner as before. Concentrations of the mixed colloids are $0.7 \times 10^{9}$ particles $\mathrm{mL}^{-1}$ and $4.1 \times 10^{9}$ particles $\mathrm{mL}^{-1}$ for the Au NSs with the diameters of $140 \mathrm{~nm}$ and $240 \mathrm{~nm}$, respectively. The ratio of the particle concentration was ca. 1:6 $(d=140 \mathrm{~nm}: d=240 \mathrm{~nm})$. In such a mixture of nanocolloids, smaller ones are difficult to be trapped selectively. In addition, because of the concentration ratio and the size dependence on scattering intensity, the spectroscopic evaluation of smaller ones would be very challenging.

Figure 7 a-d show a series of scattering images on the selective trapping and the thermophoretic depletion of the mixed nanocolloids, at the power density of $2.5 \times 10^{5} \mathrm{~W} \mathrm{~cm}^{-2}$ and the spot diameter of $3.6 \mu \mathrm{m}$. In Figure 7a, brighter Au NSs (i.e. $d=240 \mathrm{~nm}$ ) are swept away from the focal spot. On the other hand, one Au NS with low scattering intensity is trapped at the center of the image. With the passage of time, another Au NS came into the focal spot, and stably trapped (Figure 7 c-d, and see Supplementary Movie 4). Figure 7e shows the scattering spectrum of the trapped two Au NSs at the center of (a)-(d). Calculated scattering spectra of Au NSs with the diameters of $140 \mathrm{~nm}$ and $240 \mathrm{~nm}$ from (dotted and dashed lines) Mie theory are shown for comparison ${ }^{46}$ For the calculation, the core diameters $\left(d_{\text {core }}\right)$ and the shell thicknesses $\left(t_{\text {shell }}\right)$ were given as follows: $d_{\text {core }}=80 \mathrm{~nm}$ and $t_{\text {shell }}=30 \mathrm{~nm}$ for the 140 -nm-diameter Au NS, $d_{\text {core }}=200 \mathrm{~nm}$ 
and $t_{\text {shell }}=20 \mathrm{~nm}$ for the 240-nm-diameter Au NS. Refractive indices of silica and water were 1.46 and 1.33, respectively. The peak wavelength and line width of the observed spectrum are well reproduced by the calculation for $d=140 \mathrm{~nm}$. The reason for smaller Au NSs being trapped despite its small diameter is attributable to smaller $\Delta T$ at the surface leading to weaker thermophoretic force. The slight red-shift of the experimental spectrum might be ascribable to interaction between two Au NSs. ${ }^{47}$ This result on plasmonic spectroscopy indicates that a few small nano-objects were selectively trapped and their optical properties were successfully measured at the singlenanoparticle level, because bright scatterers were spatially eliminated by the thermophoresis.

Conclusions. Both the optical trapping and the thermophoresis can be induced only by irradiating colloidal solution with a focused NIR laser. Until now, the thermophoresis is considered as an undesired side effect on optical manipulation. However, we successfully demonstrated that the interplay between the optical- and the thermophoretic forces enables the selective trapping of specific single nano-objects and the spatial separation of undesired others. The most important point in the present method is the feasible design for attractive- and repulsive forces acting on nano-objects by optimizing irradiation conditions. As a result, we have succeeded in the spectroscopic characterization of specific nano-objects at single-nanoparticle levels in the dense mixture of nanocolloids. The present work paves a way for manipulation and spectroscopy of single molecules in dense and complex environments. 


\section{FIGURES}

Figure 1

(a)

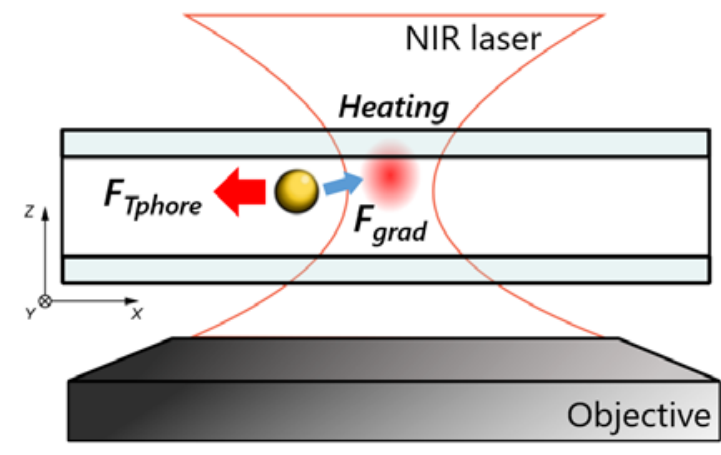

(b)

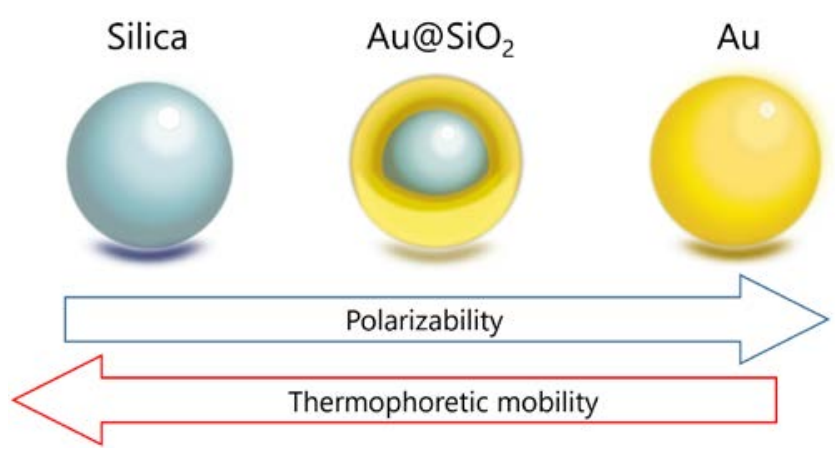

Figure 1. (a) Schematic illustration of optical gradient- and thermophoretic forces acting on a plasmonic nanoparticle under irradiation of a focused NIR laser. (b) Optical- and thermal properties of silica, $\mathrm{Au} @ \mathrm{SiO}_{2}$, and Au nanoparticles. 
Figure 2

(a)

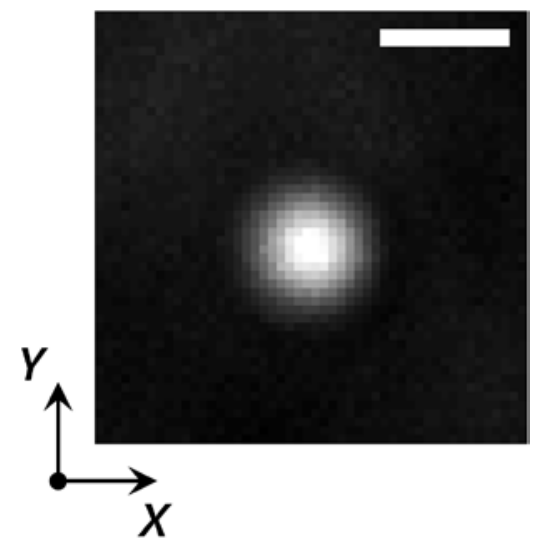

(c)

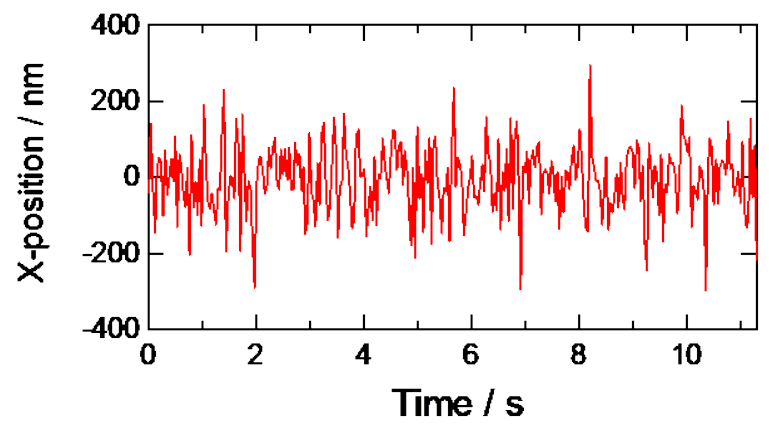

(b)

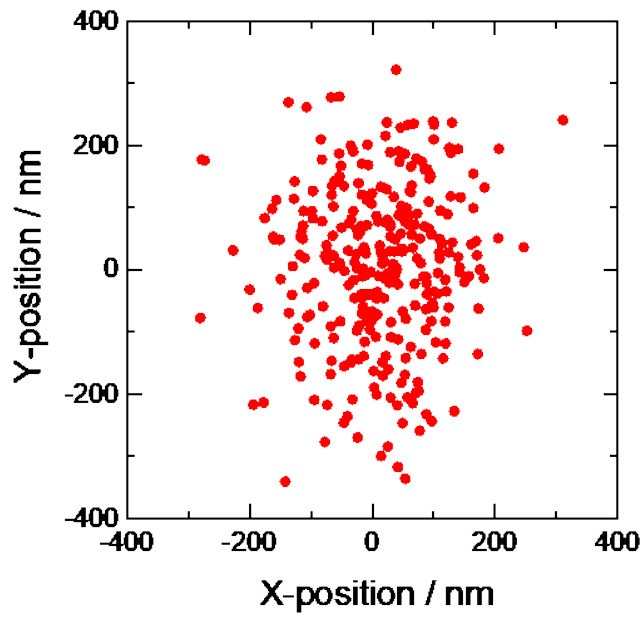

(d)

Figure 2. (a) Optical scattering image of an optically trapped single Au NS. Scale bar: $2 \mu \mathrm{m}$. (b) Distribution of the $X Y$ position of an optically trapped Au NS at a power density of $4.3 \times 10^{5} \mathrm{~W}$ $\mathrm{cm}^{-2}$. (c) Time course of positional fluctuation along the $X$ axis of the trapped Au NS. (d) 
Histogram of the $X$ position for the time trajectory shown in panel c. The solid line is the curve obtained by the analysis with a Gauss function.

Figure 3

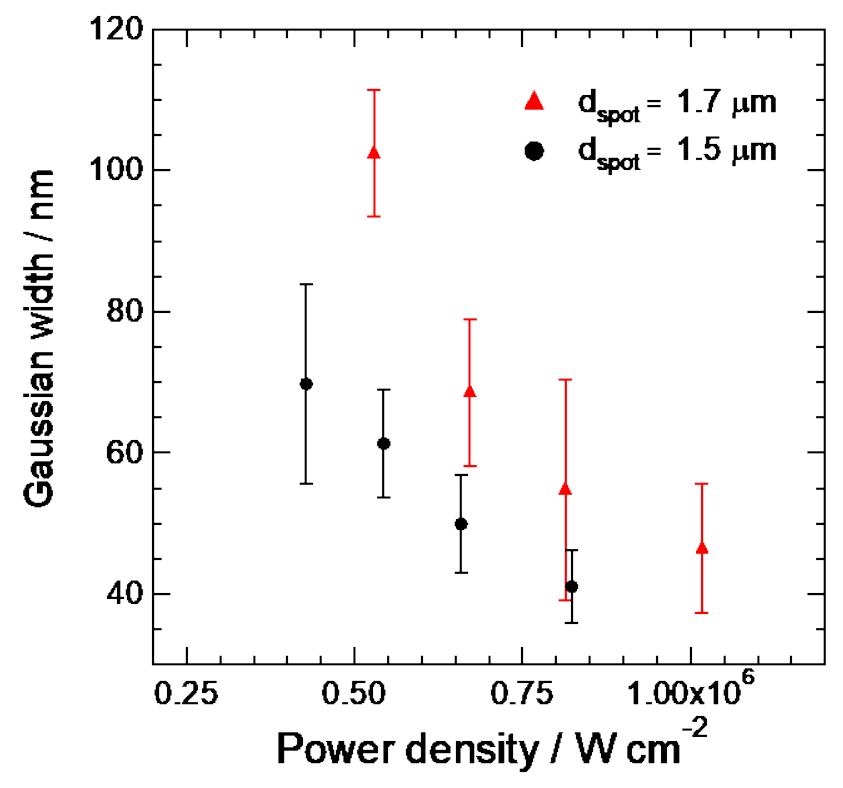

Figure 3. Positional fluctuations of a trapped Au NS as a function of power density at laser spot diameters of 1.5 and $1.7 \mu \mathrm{m}$. 
Figure 4

(a)

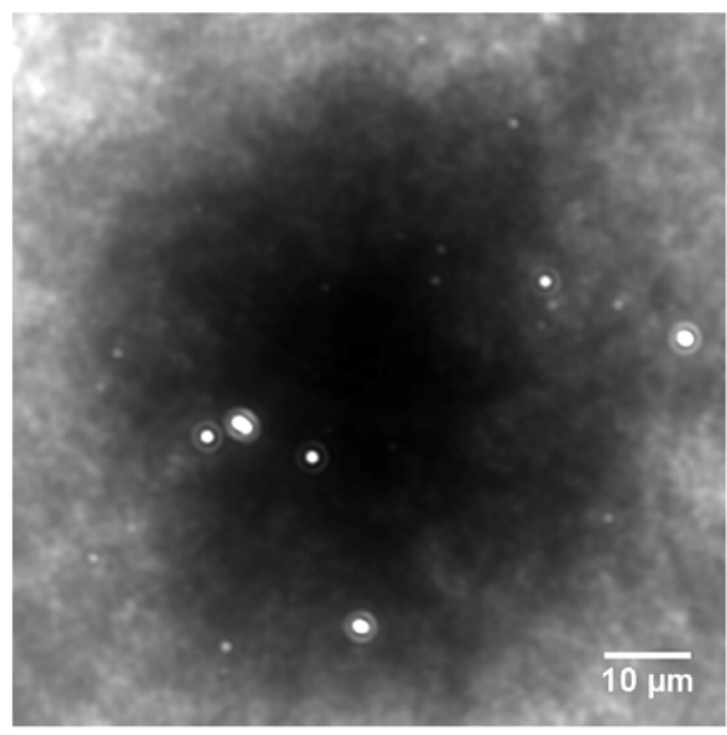

(b)

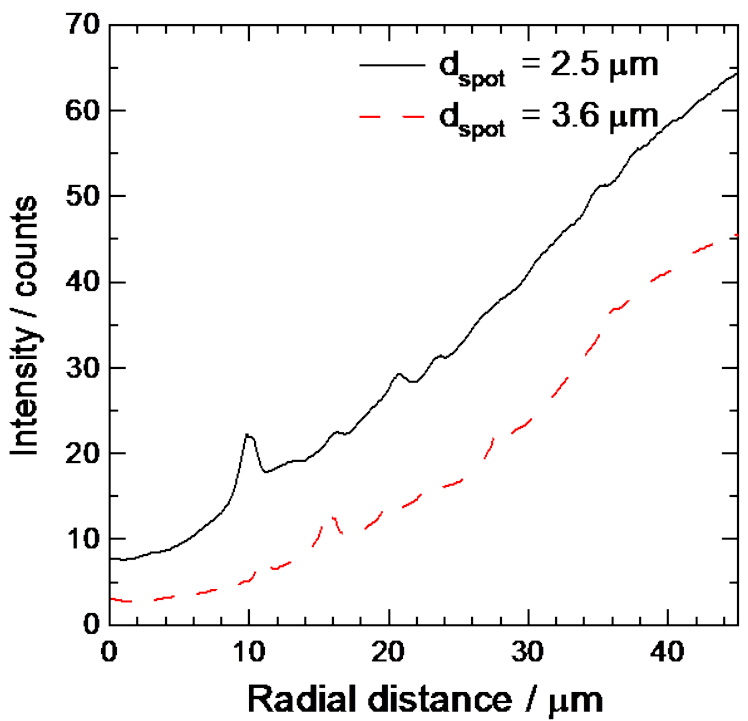

Figure 4. (a) Scattering image accumulated 100 frames under irradiation at a power density of $2.2 \times 10^{5} \mathrm{~W} \mathrm{~cm}^{-2}$ and a laser spot diameter of $3.6 \mu \mathrm{m}$. (b) Averaged intensity profiles from a focal spot at a power density of $2.2 \times 10^{5} \mathrm{~W} \mathrm{~cm}^{-2}$. 
Figure 5

(a)

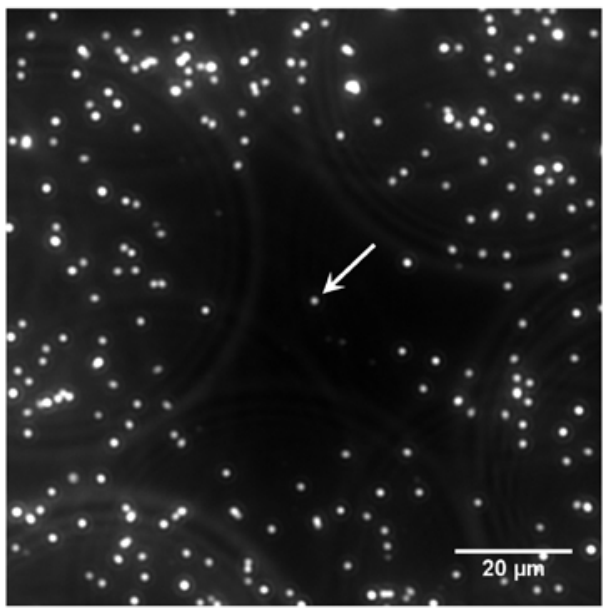

(b)

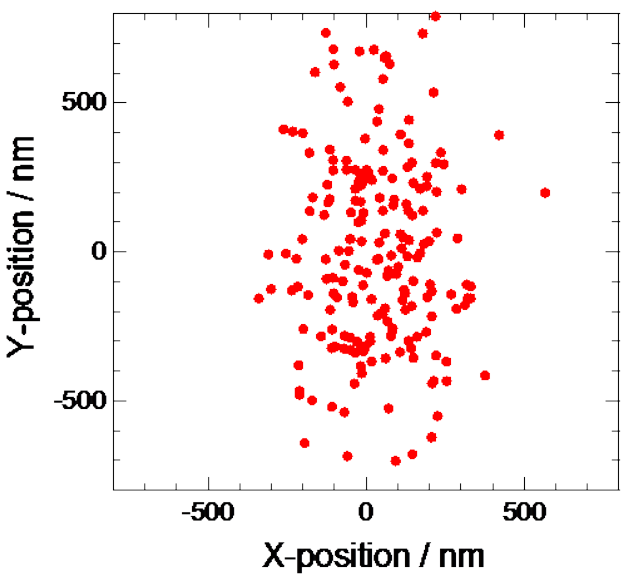

(c)

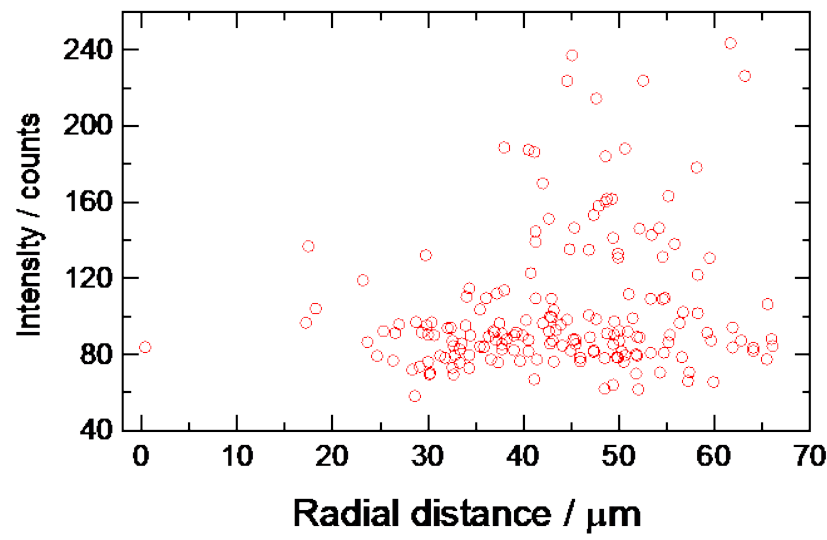

Figure 5. (a) Scattering image showing simultaneous optical trapping of a single Au NS and thermophoretic depletion of others. The trapped Au NS is highlighted by a white arrow. (b) Distribution of the $X Y$ position of a trapped Au NS at a power density of $3.2 \times 10^{5} \mathrm{~W} \mathrm{~cm}^{-2}$ and a spot diameter of $3.0 \mu \mathrm{m}$. (c) Relationship between maximum brightness of each Au NS and radial distance from a focal spot. 
Figure 6

(a)

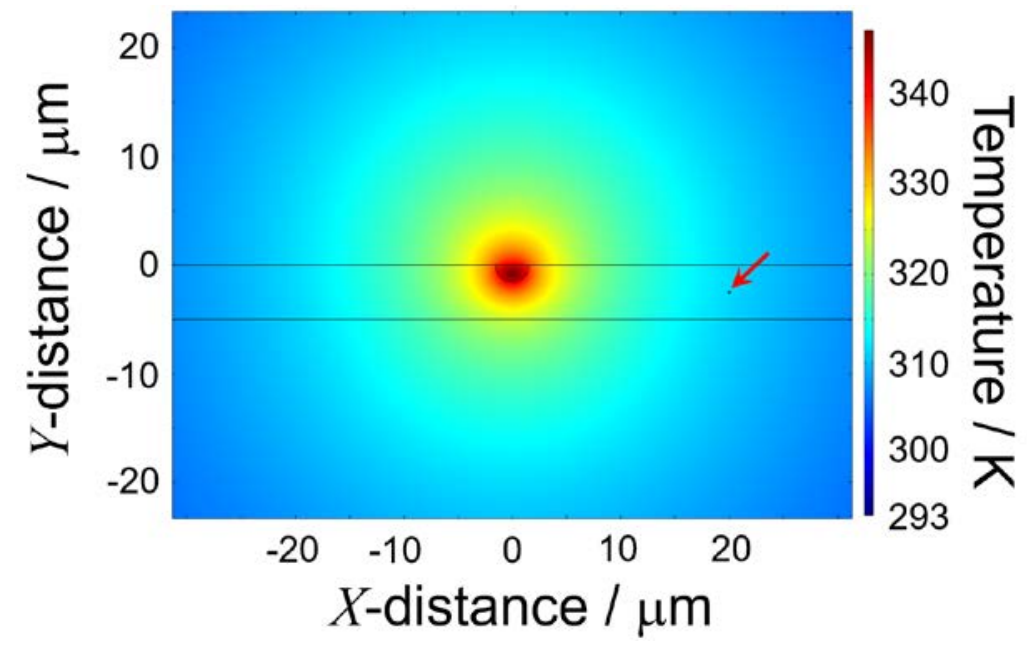

(b)

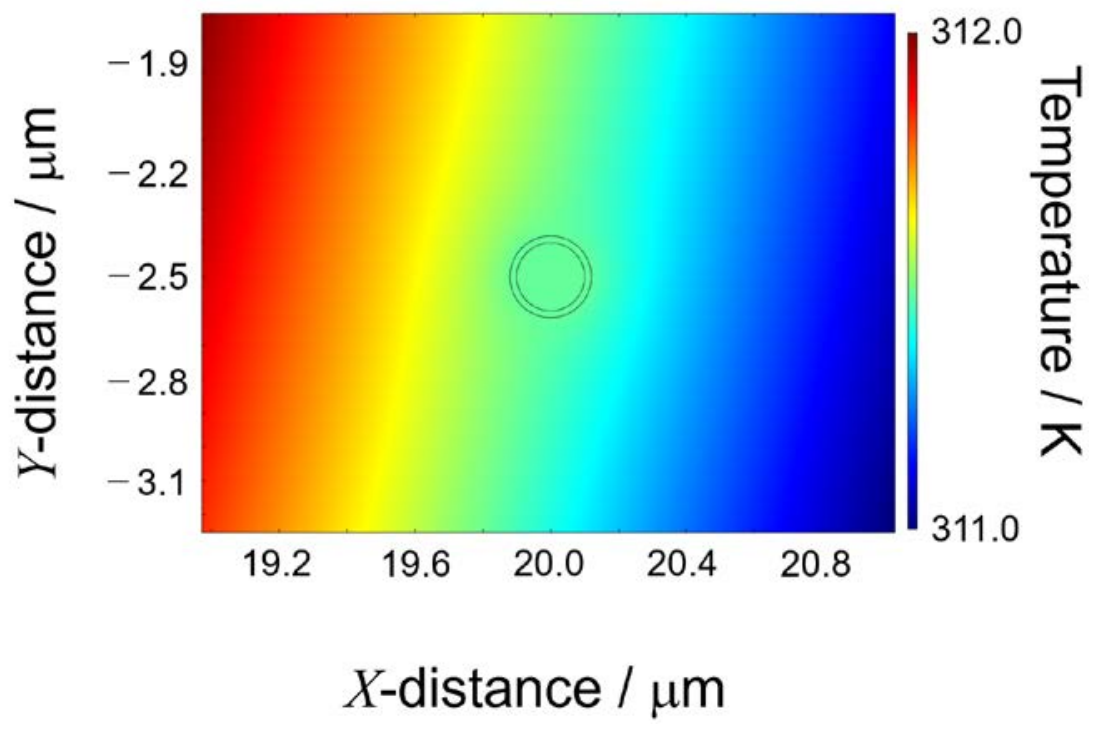

(c) 


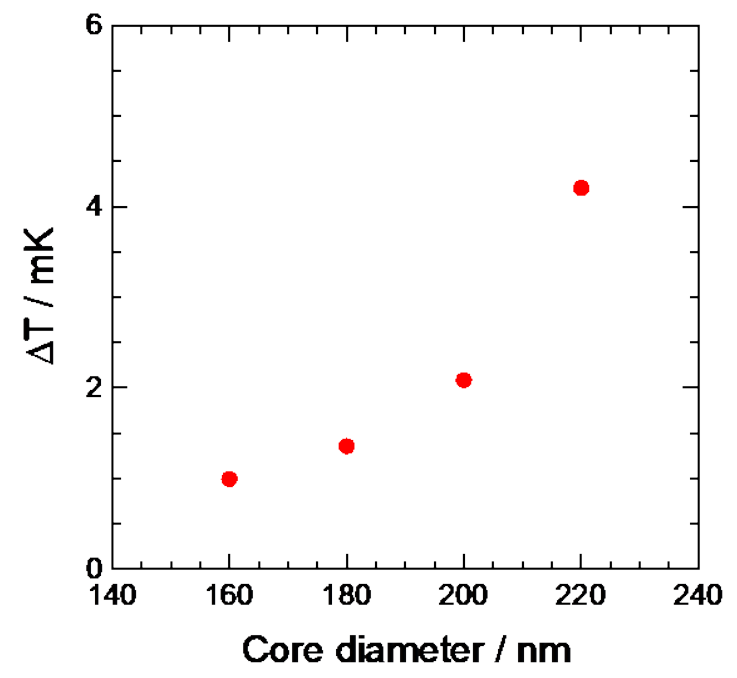

Figure 6. (a) Calculated 2D temperature distribution for the system consisting of a water layer sandwiched with two glass substrates and a Au NS. The Au NS is highlighted by a red arrow. (b) Magnified 2D temperature distribution around a Au NS. (c) Temperature difference ( $\Delta T$ ) at a surface of the Au NS as the function of core diameter at a constant core/shell diameter of 240 nm. 
Figure 7

(a)

(b)
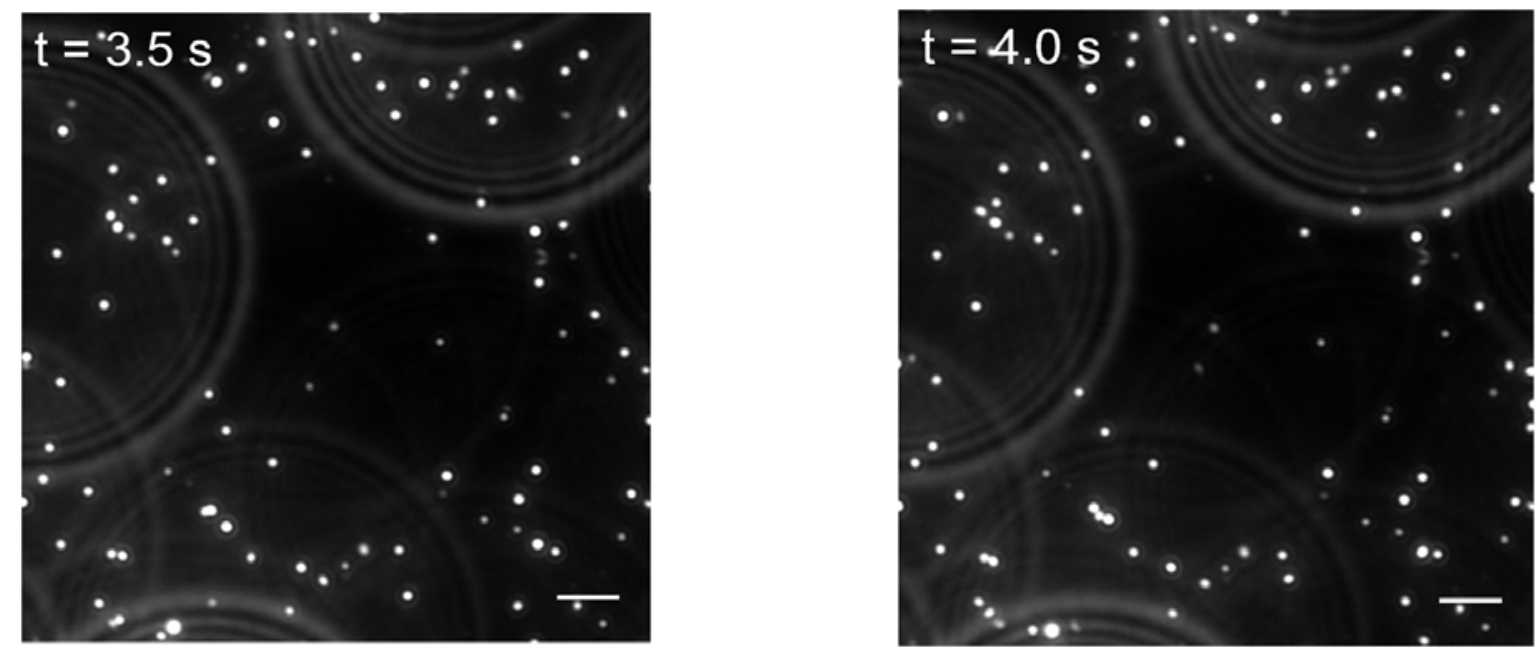

(c)

(d)
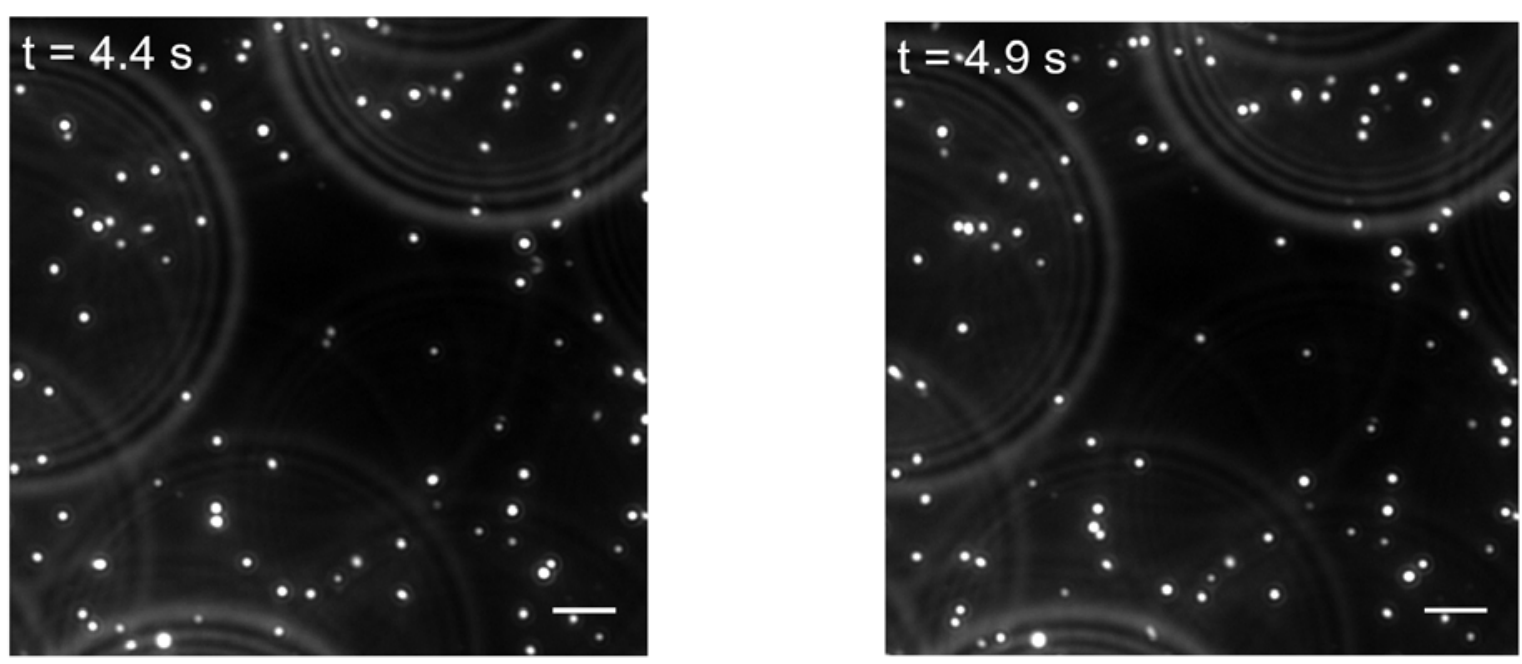
(e)

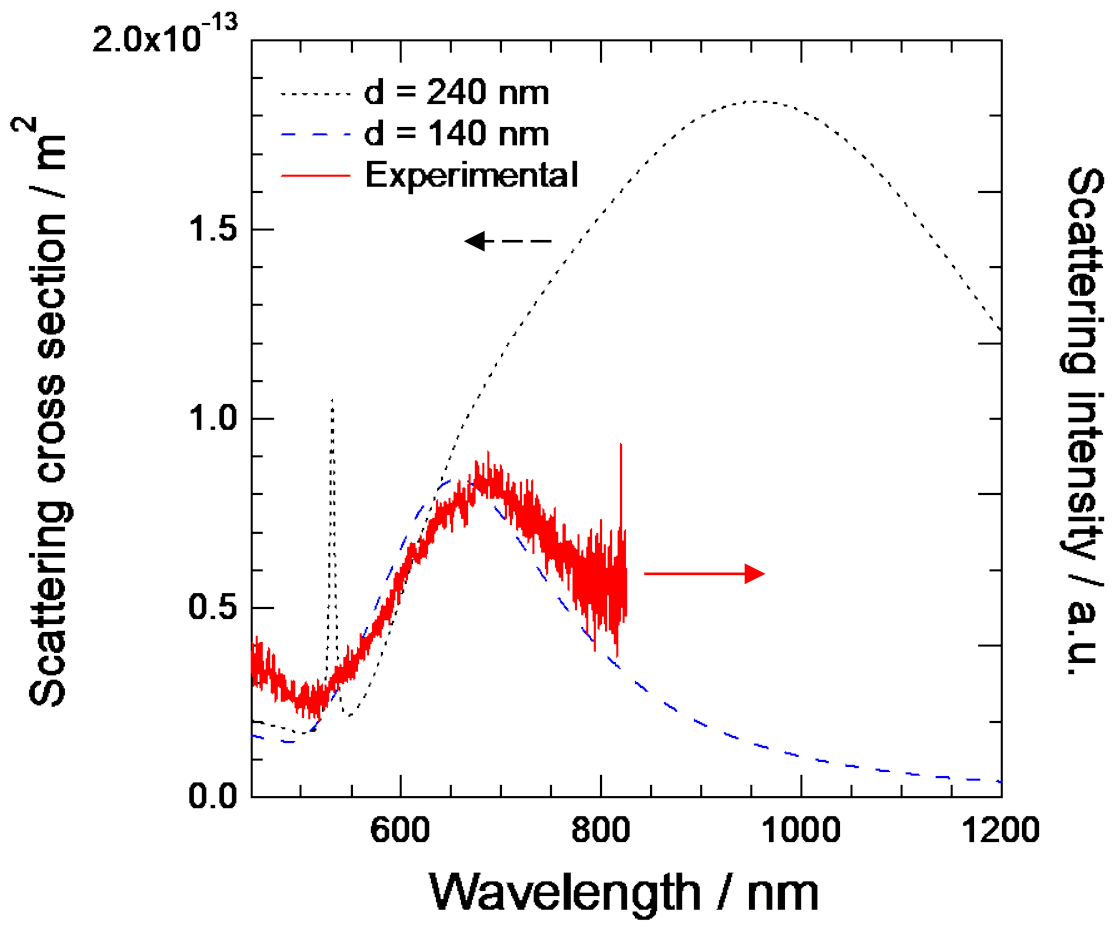

Figure 7. (a)-(d) Time course of scattering images of simultaneous optical trapping of a few Au NSs with a diameter of $140 \mathrm{~nm}$ and thermophoretic depletion of Au NSs with diameters about $240 \mathrm{~nm}$. Scale car: $10 \mu \mathrm{m}$. (e) Scattering spectrum of a few trapped Au NSs at the center of (a)(d). Calculated scattering spectra of a Au NS with diameters of 140 and $240 \mathrm{~nm}$ (dotted and dashed lines) are shown for comparison. 


\section{ASSOCIATED CONTENT}

Electronic Supplementary Information (ESI). Optical setup (Figure S1), photoluminescent image of the focused laser (Figure S2), histograms of the optically trapped Au NS (Figure S3), temperature calculation of the laser spot (Figure S4), and numerical calculations of the thermophoretic- and the Stoke drag force (PDF).

Supplementary movie 1: Optical trapping of a Au NS (AVI)

Supplementary movie 2: Dynamics of thermophoretic depletion (AVI)

Supplementary movie 3: Trapping and Sorting of Au NSs with a diameter of $240 \mathrm{~nm}$ (AVI)

Supplementary movie 4: Selective trapping and separation of Au NSs with diameters of $140 \mathrm{~nm}$ and $240 \mathrm{~nm}(\mathrm{AVI})$

\section{AUTHOR INFORMATION}

\section{Corresponding Authors}

*E-mail: setoura@es.hokudai.ac.jp

*E-mail: sito@chem.es.osaka-u.ac.jp

\section{ORCID}

Kenji Setoura: 0000-0002-4610-7407

Tetsuro Tsuji: 0000-0002-2087-5459

Syoji Ito: 0000-0003-0582-4108

Hiroshi Miyasaka: 0000-0002-6020-6591

Present Addresses

\# Graduate School of Informatics, Kyoto University, Kyoto 606-8501, Japan. 


\section{Author Contributions}

The manuscript was written through contributions of all authors. All authors have given approval to the final version of the manuscript.

\section{Notes}

The authors declare no competing financial interest.

\section{ACKNOWLEDGMENT}

This work was supported by JSPS KAKENHI grant numbers JP16H06505 and JP16H06504 in Scientific Research on Innovative Areas “Nano-Material Optical-Manipulation”, JP26107002 in Scientific Research on Innovative Areas “Photosynergetics”, JP18K13688, JP18K13687, JP18K19057, and JP18H05242.

\section{REFERENCES}

1 A. Ashkin, J. M. Dziedzic, J. E. Bjorkholm and S. Chu, Opt. Lett., 1986, 11, 288-290.

2 A. Ashkin, IEEE J. Sel. Top. Quantum Electron., 2000, 6, 841-856.

3 T. Iida and H. Ishihara, Phys. Rev. Lett., 2003, 90, 057403.

4 K. Inaba, K. Imaizumi, K. Katayama, M. Ichimiya, M. Ashida, T. Iida, H. Ishihara and T. Itoh, Phys. Status Solidi Basic Res., 2006, 243, 3829-3833.

$5 \quad$ S. E. Skelton Spesyvtseva, S. Shoji and S. Kawata, Phys. Rev. Appl., 2015, 3, 044003.

6 S. Ito, H. Yamauchi, M. Tamura, S. Hidaka, H. Hattori, T. Hamada, K. Nishida, S. Tokonami, T. Itoh, H. Miyasaka and T. Iida, Sci. Rep., 2013, 3, 3047.

$7 \quad$ P. Juris, F. Svedberg, M. Käll, E. Jonas, R. Kerstin, G. Mattias and D. Hanstorp, Nano Lett., 2004, 4, 115-118.

$8 \quad$ Y. Pang and R. Gordon, Nano Lett., 2012, 12, 402-406. 
$9 \quad$ J. T. Finer, R. M. Simmons and J. A. Spudich, Nature, 1994, 368, 113-119.

10 A. Ishijima, H. Kojima, T. Funatsu, M. Tokunaga, H. Higuchi, H. Tanaka and T. Yanagida, Cell, 1998, 92, 161-171.

11 R. Piazza and A. Parola, J. Phys. Condens. Matter, 2008, 20, 153102.

12 A. Würger, Reports Prog. Phys., 2010, 73, 126601.

13 S. Ito, T. Sugiyama, N. Toitani, G. Katayama and H. Miyasaka, J. Phys. Chem. B, 2007, 111, 2365-2371.

14 M. Braun and F. Cichos, ACS Nano, 2013, 7, 11200-11208.

15 J. Gargiulo, T. Brick, I. L. Violi, F. C. Herrera, T. Shibanuma, P. Albella, F. G. Requejo, E. Cortés, S. A. Maier and F. D. Stefani, Nano Lett., 2017, 17, 5747-5755.

16 L. Lin, M. Wang, X. Peng, E. N. Lissek, Z. Mao, L. Scarabelli, E. Adkins, S. Coskun, H. E. Unalan, B. A. Korgel, L. M. Liz-Marzán, E.-L. Florin and Y. Zheng, Nat. Photonics, 2018, 12, 195-201.

17 L. Lin, E. H. Hill, X. Peng and Y. Zheng, Acc. Chem. Res., 2018, 51, 1465-1474.

18 M. Braibanti, D. Vigolo and R. Piazza, Phys. Rev. Lett., 2008, 100, 108303.

19 H. Misawa, M. Koshioka, K. Sasaki, N. Kitamura and H. Masuhara, Chem. Lett., 1991, 20, 469-472.

20 M. P. MacDonald, G. C. Spalding and K. Dholakia, Nature, 2003, 426, 421-424.

21 S. J. Oldenburg, J. B. Jackson, S. L. Westcott and N. J. Halas, Appl. Phys. Lett., 1999, 75, 2897.

22 T. Tsuji, S. Saita and S. Kawano, Phys. Rev. Appl., 2018, 9, 024035.

23 T. Tsuji, Y. Sasai and S. Kawano, Phys. Rev. Appl., 2018, 10, 044005.

24 Y. Pang and R. Gordon, Nano Lett., 2011, 11, 3763-3767. 
25 F. Hajizadeh and S. N. S.Reihani, Opt. Express, 2010, 18, 551.

26 A. P. Bregulla, A. Würger, K. Günther, M. Mertig and F. Cichos, Phys. Rev. Lett., 2016, 116, 188303.

27 P. M. Bendix, S. N. S. Reihani and L. B. Oddershede, ACS Nano, 2010, 4, 2256-2262.

28 S. Ito, M. Mitsuishi, K. Setoura, M. Tamura, T. Iida, M. Morimoto, M. Irie and H. Miyasaka, J. Phys. Chem. Lett., 2018, 9, 2659-2664.

29 K. Setoura, K. Fujita, S. Ito and H. Miyasaka, J. Nanophotonics, 2018, 13, 012504.

30 M. Braun, A. P. Bregulla, K. Günther, M. Mertig and F. Cichos, Nano Lett., 2015, 15, 5499-5505.

31 E. J. G. Peterman, F. Gittes and C. F. Schmidt, Biophys. J., 2003, 84, 1308-1316.

32 Y. Seol, A. E. Carpenter and T. T. Perkins, Opt. Lett., 2006, 31, 2429-2431.

33 G. Baffou, J. Polleux, H. Rigneault and S. Monneret, J. Phys. Chem. C, 2014, 118, 48904898.

34 Y. Xie and C. Zhao, Nanoscale, 2017, 9, 6622-6631.

35 L. Lin, X. Peng, Z. Mao, W. Li, M. N. Yogeesh, B. B. Rajeeva, E. P. Perillo, A. K. Dunn, D. Akinwande and Y. Zheng, Nano Lett., 2016, 16, 701-708.

36 A. Miniewicz, C. Quintard, H. Orlikowska and S. Bartkiewicz, Phys. Chem. Chem. Phys., 2017, 19, 18695-18703.

37 G. M. Hale and M. R. Querry, Appl. Opt., 1973, 12, 555-563.

38 K. Setoura, S. Ito and H. Miyasaka, Nanoscale, 2017, 9, 719-730.

39 S. Duhr and D. Braun, Phys. Rev. Lett., 2006, 96, 168301.

40 H. R. Jiang, H. Wada, N. Yoshinaga and M. Sano, Phys. Rev. Lett., 2009, 102, 208301. 
41 X. Lu, M. Rycenga, S. E. Skrabalak, B. Wiley and Y. Xia, Annu. Rev. Phys. Chem., 2009, 60, 167-192.

42 K. Setoura, Y. Okada, D. Werner and S. Hashimoto, ACS Nano, 2013, 7, 7874-7885.

43 P. Keblinski, D. G. Cahill, A. Bodapati, C. R. Sullivan and T. A. Taton, J. Appl. Phys., 2006, 100, 054305.

44 G. Baffou and H. Rigneault, Phys. Rev. B - Condens. Matter Mater. Phys., 2011, 84, 035415.

45 J. Januszewski, M. I. Khokhar and A. S. Mujumdar, Lett. Heat Mass Transf., 1977, 4, 417-423.

46 K. Setoura, D. Werner and S. Hashimoto, J. Phys. Chem. C, 2012, 116, 15458-15466.

47 N. J. Halas, S. Lal, W.-S. Chang, S. Link and P. Nordlander, Chem. Rev., 2011, 111, 3913-3961. 